SUBJECT AREAS:

TWO-DIMENSIONAL MATERIALS

ELECTRONIC DEVICES

Received

28 October 2014

Accepted

24 December 2014

Published

21 January 2015

Correspondence and requests for materials should be addressed to

S.-L.Z. (shili.zhang@ angstrom.uu.se); R.L. (rliu@fudan.edu.cn) or Z.J.Q. (ziqiu@fudan. edu.cn)

\section{Photothermoelectric and photovoltaic effects both present in $\mathrm{MoS}_{2}$}

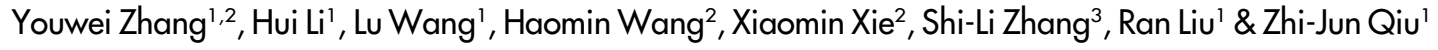

'State Key Laboratory of ASIC \& System, School of Information Science and Technology, Fudan University, Shanghai 200433 China, ${ }^{2}$ State Key Laboratory of Functional Materials for Informatics, Shanghai Institute of Microsystem \& Information Technology, Chinese Academy of Sciences, Changning Road 865, Shanghai 200050, China, ${ }^{3}$ Solid-State Electronics, The Ångström Laboratory, Uppsala University, Uppsala Box 534, SE-751 21, Sweden.

As a finite-energy-bandgap alternative to graphene, semiconducting molybdenum disulfide $\left(\mathrm{MoS}_{2}\right) \mathrm{has}$ recently attracted extensive interest for energy and sensor applications. In particular for broad-spectral photodetectors, multilayer $\mathrm{MoS}_{2}$ is more appealing than its monolayer counterpart. However, little is understood regarding the physics underlying the photoresponse of multilayer $\mathrm{MoS}_{2}$. Here, we employ scanning photocurrent microscopy to identify the nature of photocurrent generated in multilayer $\mathrm{MoS}_{2}$ transistors. The generation and transport of photocurrent in multilayer $\mathrm{MoS}_{2}$ are found to differ from those in other low-dimensional materials that only contribute with either photovoltaic effect (PVE) or photothermoelectric effect (PTE). In multilayer $\mathrm{MoS}_{2}$, the PVE at the $\mathrm{MoS}_{2}$-metal interface dominates in the accumulation regime whereas the hot-carrier-assisted PTE prevails in the depletion regime. Besides, the anomalously large Seebeck coefficient observed in multilayer $\mathrm{MoS}_{2}$, which has also been reported by others, is caused by hot photo-excited carriers that are not in thermal equilibrium with the $\mathrm{MoS}_{2}$ lattice.

T wo-dimensional materials have lately attracted tremendous attention owing to their unique structural and physical properties ${ }^{1}$. Understanding the basic physical phenomena as well as their intimate interplays in this new family of materials is essential for further exploitation of the materials towards electronic, photonic, and energy applications. Although it is largely graphene's tribute that has sparked the recent zeal in two-dimensional materials and their potential applications, the intrinsic shortcoming without a bandgap hampers its application in electronics ${ }^{2}$. For this reason, attention has been turned to other two-dimensional semiconducting transition metal dichalcogenides (TMDs) for their potential role in nanoelectronics, spintronics and optoelectronics ${ }^{3,4}$. Among them, molybdenum disulfide $\left(\mathrm{MoS}_{2}\right)$, as a representative layered TMD, has a layer-dependent bandgap with a crossover from an indirect bandgap of $1.3 \mathrm{eV}$ in its multilayer form to a direct bandgap of $1.8 \mathrm{eV}$ when thinned to a monolayer ${ }^{5,6}$. Both single- and multi-layer $\mathrm{MoS}_{2}$ have been demonstrated to be suitable for electronic devices, such as field-effect transistors $(\mathrm{FETs})^{7,8}$, photodetectors ${ }^{9,10}$, gas sensors ${ }^{11}$, etc. However, multilayer $\mathrm{MoS}_{2}$ is of greater potential than the monolayer counterpart from the fabrication viewpoint as well as the interesting physical properties. First, the electronic properties of multilayer $\mathrm{MoS}_{2}$ manifested by an indirect bandgap are relatively insensitive to layer thickness. Second, multilayer $\mathrm{MoS}_{2}$ is expected to carry higher drive current than monolayer $\mathrm{MoS}_{2}$ due to higher carrier mobility arising from interlayer screening effect ${ }^{12}$ and the 3 -fold higher density of states at the conduction band minimum ${ }^{8,13}$.

With respect to optical properties, the subnanometer thickness of monolayer $\mathrm{MoS}_{2}$ yields a low absorption cross-section hence results in less photo-generated carriers, as compared to that of multilayer $\mathrm{MoS}_{2}$. Although fast photodetection has been achieved in monolayer-MoS transistors $^{14}$, the photoresponsivity is expected to be lower than that of multilayer-MoS devices $^{10}$, due to its direct bandgap and resultant shorter photocarrier recombination lifetime $(\sim 70 \mathrm{ps})^{15}$. For multilayer $\mathrm{MoS}_{2}$, intervalley scattering is predominant over direct electron-hole (e$\mathrm{h}$ ) recombination when incident light energy is larger than direct bandgap at $\mathrm{K}$ point, thus generating microsecond-order photocarrier lifetime of indirect recombination ${ }^{16}$. This was further proven by quenching of photoluminescence in multilayer $\mathrm{MoS}_{2}{ }^{6}$. The long carrier lifetime would have a positive effect on photoresponsivity. There is therefore greater potential with multilayer $\mathrm{MoS}_{2}$ than monolayer $\mathrm{MoS}_{2}$ for applications in broad-spectral photodetectors.

Investigations of multilayer $\mathrm{MoS}_{2}$ and other TMD semiconductors are scarce and insufficient, compared to their monolayer counterparts, especially on optoelectronic properties. The origin of the observed photoresponse in $\mathrm{MoS}_{2}$ is still under debate. Most studies suggest that photocurrent generated in the $\mathrm{MoS}_{2}$ phototransistors results from photovoltaic effect (PVE) $)^{9,10,14,17,18}$, similarly to what has been found with other conventional 
semiconductors, e.g. Si, GaAs and GaN. Another photocurrent mechanism, i.e. photothermoelectric effect (PTE), is less discussed in the literature, in part due to the use of a broad-beam illumination experimental setup. Recent studies on graphene $e^{19,20}$ and carbon nanotubes ${ }^{21,22}$ indicate that PTE may play an important role in their photoresponse. As the amplitude of PTE relies on the temperature difference, multilayer $\mathrm{MoS}_{2}$ with a well-defined bulk, and therefore a reduced thermal sinking effect via the substrate, is expected to yield a higher temperature increase under illumination ${ }^{23}$. Because of the complex nature of $\mathrm{MoS}_{2}$, there is need to locally probe its photoresponse in order to allow the mechanisms of charge transport and collection in multilayer $\mathrm{MoS}_{2}$ to be unequivocally unveiled and, in particular, to discern PTE from PVE.

In order to elucidate the nature of photoresponse in thick $\mathrm{MoS}_{2}$ multilayers, we have employed scanning photocurrent microscopy (SPCM) in combination with ordinary current-voltage characterization to characterize the spatial dependence of photoresponse under various gate bias $\left(V_{\mathrm{g}}\right)$ conditions. At the metal-semiconductor interface, PVE is found to be predominant in the accumulation regime while thermal diffusion of photo-excited hot electrons is overwhelming and dictates the drifting current by the built-in electric field in the depletion regime.

\section{Results}

Device structure and characterization. The schematic representation of a back-gate $\mathrm{MoS}_{2}$ transistor used in our work is shown in Fig. 1a while its optical microscope image is given in Fig. 1b. Isolated $\mathrm{MoS}_{2}$ flakes on a $\mathrm{SiO}_{2} / \mathrm{Si}$ substrate were exfoliated from a bulk $\mathrm{MoS}_{2}$ crystal using a conventional mechanical exfoliation technique ${ }^{24}$. The sample preparation and device fabrication are detailed in Methods. The thickness of the $\mathrm{MoS}_{2}$ flake in the device in Fig. 1b, as measured by atomic force microscopy (AFM), is approximately $65 \mathrm{~nm}$ (Fig. 1c) and its Raman spectrum in Fig. 1d shows two typical peaks $\left(\mathrm{E}_{2 \mathrm{~g}}{ }^{1}\right.$ and $\left.\mathrm{A}_{1 \mathrm{~g}}\right)$

a
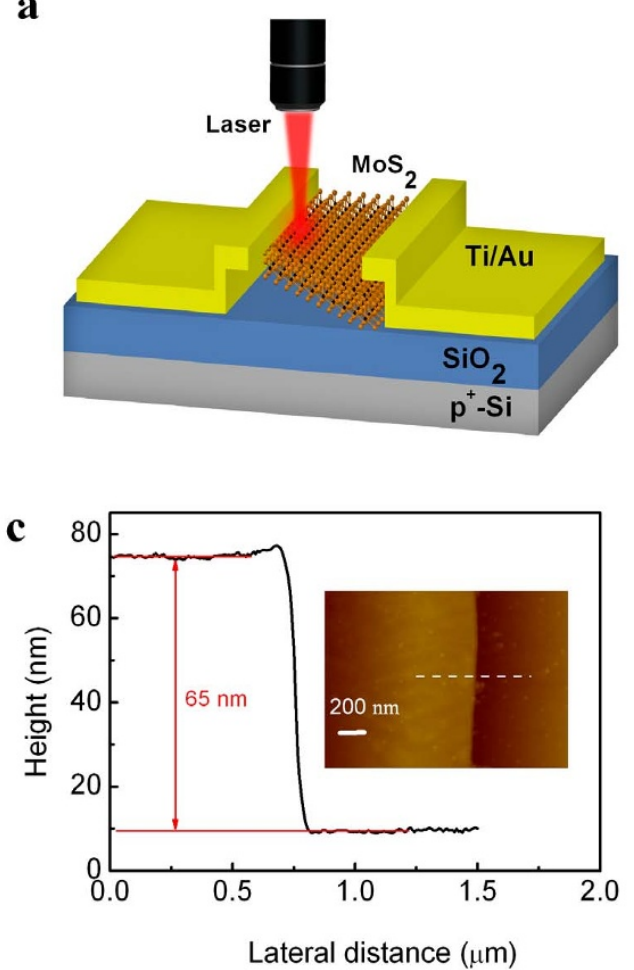

with a large separation of $25 \mathrm{~cm}^{-1}$, confirming that the multilayer nature of the $\mathrm{MoS}_{2}$ flake'.

Photoelectrical stability of $\mathbf{M o S}_{\mathbf{2}}$ transistor. Electrical characterization of our $\mathrm{MoS}_{2}$ FETs was performed in ambient environment. Similar to other low-dimensional devices without surface protection ${ }^{26-28}, \mathrm{MoS}_{2}$ FETs are highly sensitive to extrinsic effects, especially adsorption of $\mathrm{H}_{2} \mathrm{O} / \mathrm{O}_{2}$ from air $^{29,30}$. These effects can induce current hysteresis in transfer characteristics and electrical stress instability of $\mathrm{MoS}_{2}$ FETs when measured in conventional DC mode (Supplementary Fig. S1). The electrical instability can lead to persistent photoconductivity when switching on and off the laser illumination and therefore, a reduction of photoresponsivity with switching time (Supplementary Figs. S3 and S4). Therefore, stability of the device under electrical stress is a prerequisite for practical optoelectronic applications of $\mathrm{MoS}_{2}$. Recently, electrical characterization with $V_{\mathrm{g}}$ pulses of alternating polarities i.e. AP mode, has been reported to distinctively reduce the device instability compared to using DC mode $e^{28,31,32}$. As shown in Supplementary Fig. S1 and Fig. 2a, the hysteresis of our $\mathrm{MoS}_{2}$ FETs is almost completely eliminated and stable electrical characteristics are obtained using the AP method. This method leads indeed to stable photoswitching behaviors when applying multiple illuminations (Fig. 2b and Supplementary Fig. S4), which can only be achieved in vacuum if $\mathrm{MoS}_{2}$ FETs are measured using the DC method ${ }^{33}$. Since the AP method is not based on any structural or chemical modification of the devices, as opposed to other measures employed to improve the device stability ${ }^{26,29,34}$, it is of great potential in leading to more repeatable and clearly interpretable photo-sensing signals. Therefore, all our photoelectrical measurements were performed in AP mode in order to avoid additional stressing of the devices.

SPCM characterization of $\mathrm{MoS}_{2}$ transistor. To identify the mechanism of photocurrent generation, SPCM was conducted at zero source-drain bias $\left(V_{\mathrm{d}}=0 \mathrm{~V}\right)$ with a well-focused laser beam

b

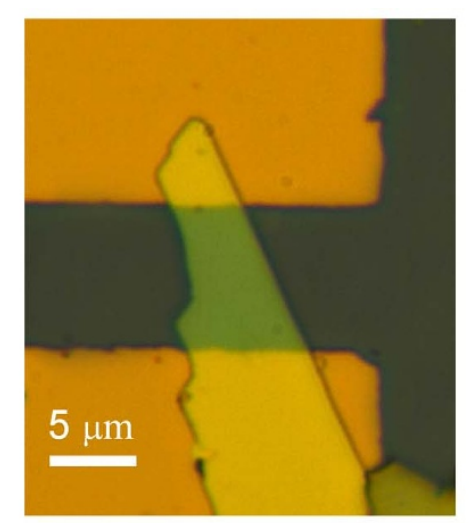

d

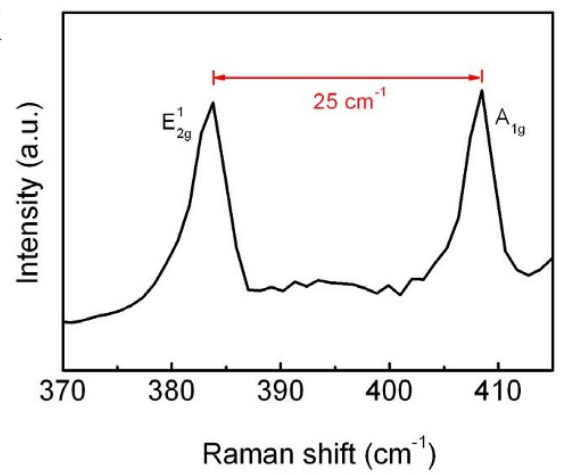

Figure $1 \mid$ Device structure and characterization. (a) Schematic and (b) Optical image of the field-effect transistor based on multilayer MoS 2 . (c) AFM line scan along the dashed line across the boundary of the $\mathrm{MoS}_{2}$ flake in the inset. Inset: high-resolution AFM image of the MoS $\mathrm{S}_{2}$ multilayer on $\mathrm{SiO}_{2} / \mathrm{Si}$ substrate. (d) Raman spectrum for the $\mathrm{MoS}_{2}$ multilayer on $\mathrm{SiO}_{2} / \mathrm{Si}$ substrate. The frequency separation between $\mathrm{E}_{2 \mathrm{~g}}$ and $\mathrm{A}_{1 \mathrm{~g}}$ peaks is $25 \mathrm{~cm}^{-1}$. 

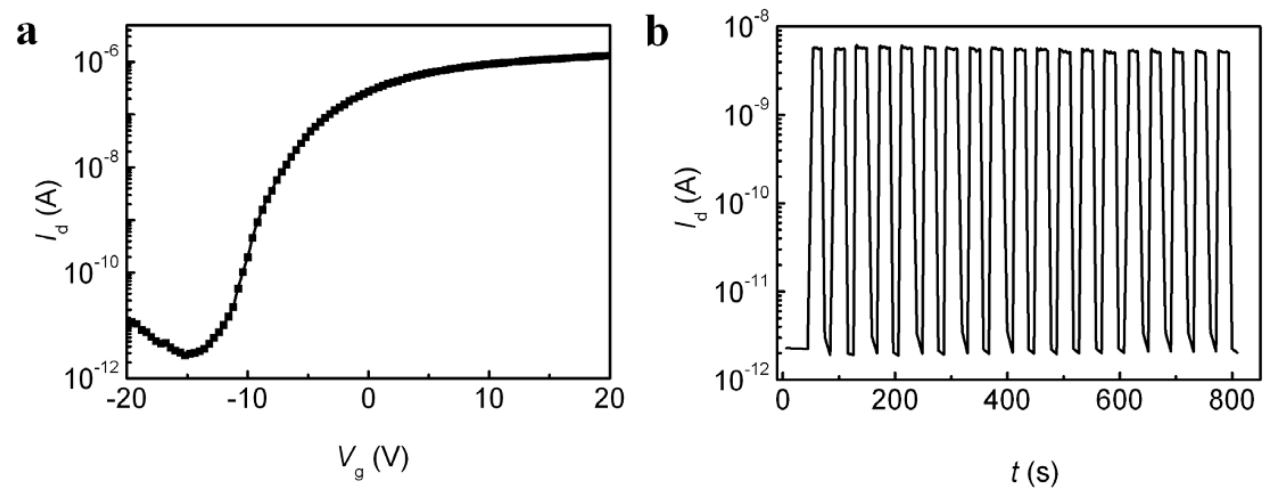

Figure $2 \mid$ Electrical characteristics and photoswitching behavior of $\mathrm{MoS}_{2}$ transistor. (a) Transfer characteristics of the $\mathrm{MoS}_{2}$ transistor, measured at $V_{\mathrm{d}}$ $=1 \mathrm{~V}$, with $V_{\mathrm{g}}$ sweeping in AP mode. (b) Stability of photoswitching behavior of the $\mathrm{MoS}_{2}$ transistor at $V_{\mathrm{d}}=1 \mathrm{~V}$ and $V_{\mathrm{g}}=-15 \mathrm{~V}$ in AP mode.

The laser spot was focused in the $\mathrm{MoS}_{2}$ channel with a laser power of $100 \mu \mathrm{W}$.

scanning over the device area. The short-circuit photocurrent and Raman spectra were recorded simultaneously as a function of laser position. To avoid possible photo-damage to the $\mathrm{MoS}_{2}$ during experiment $^{35}$, the step size was set to be $1 \mu \mathrm{m}$. Figure $3 \mathrm{a}$ shows the photocurrent image measured at $V_{\mathrm{g}}=15 \mathrm{~V}$, where two photocurrent extrema of opposite polarities are observed at the electrode edges. As expected from conventional PVE, the opposite built-in electric fields from space charges at the source/drain contacts create an asymmetrical photocurrent characteristic. Such PVEinduced photocurrents localized near electrodes have been widely reported in devices based on silicon nanowires ${ }^{36}$ and carbon nanotubes ${ }^{37,38}$. However, it is remarkable to observe a significant photocurrent when the laser spot is centered inside the electrodes. The distance from the electrode edge is $\sim 10$ times larger than the laser spot size in order to make sure that the $\mathrm{MoS}_{2}$ channel is not illuminated. Besides, the light penetration depth is smaller than the thickness for the metal electrodes at the wavelengths used ${ }^{39}$. The absence of Raman signals from the $\mathrm{MoS}_{2}$ flake under the Ti/Au electrodes in Fig. 3b implies, therefore, that the observed photocurrent inside the electrodes could not arise from PVE at the $\mathrm{MoS}_{2}$-metal interface under the electrodes. Another photocurrent mechanism than PVE should be at work.

In fact, the absorption of laser photo energy by the metal electrodes in this manner could result in an inhomogeneous local temperature distribution. When a temperature gradient is generated by laser heating across a junction comprising two materials of different Seebeck coefficients, a thermal voltage driving a current through the device is established via thermoelectric effects. The polarity of thermoelectric current relies on the majority carriers (i.e. electrons or holes) and reverses sign in between the source and drain electrodes. As shown in Fig. $3 \mathrm{a}$, the photocurrent image in the electrode area coincides with the optical surface topography of the $\mathrm{MoS}_{2}$ flake underneath the metal electrodes. This suggests that the PTE mechanism cannot be ignored in the photocurrent contribution. And the PTE-induced photocurrent is also expected to be intensified at the electrode edges where light can be absorbed by both materials and the heat dissipation is lower at the electrode edge than inside the metal electrodes.

The temperature rise at the $\mathrm{MoS}_{2}-\mathrm{Ti} / \mathrm{Au}$ interface under laser illumination can be measured by Raman spectroscopy. Since the positions of the Raman-active phonons of $\mathrm{MoS}_{2}$ are sensitive to temperature $e^{40,41}$, the local temperature change induced by laser heating can be quantified. For calibration, the laser power was minimized in order to avoid heating effects in the Raman measurement and the temperature of the whole device was tuned by a temperature controller. The temperature-dependent and laser-power-dependent shifts of the Raman mode $\mathrm{E}_{2 \mathrm{~g}}{ }^{1}$ are plotted in Supplementary Fig. S5. The linear temperature coefficient for mode $\mathrm{E}_{2 \mathrm{~g}}{ }^{1}$ is extracted to be $-0.015 \mathrm{~cm}^{-1}$ / $\mathrm{K}$ in Supplementary Fig. S5c. In combination with the power-depend- ent coefficient of $-0.74 \mathrm{~cm}^{-1} / \mathrm{mW}$ (Supplementary Fig. S5d), the temperature rise in the illuminated spot can be acquired. From this measurement, a $100-\mu \mathrm{W}$ laser illumination at the $\mathrm{MoS}_{2}-\mathrm{Ti} / \mathrm{Au}$ contact could result in a temperature increase by $\sim 5 \mathrm{~K}$. Thicker $\mathrm{MoS}_{2}$ layer could lead to a higher temperature rise due to the reduction of thermal coupling to the substrate ${ }^{23}$.

Photothermoelectric and photovoltaic effects. In the following discussion, we focus on two main competing mechanisms of photocurrent generation: PVE and PTE. The energy band diagrams of a $\mathrm{MoS}_{2}$ transistor with a Schottky barrier for various $V_{\mathrm{g}}$ conditions are schematically shown in Fig. 3c. For large positive $V_{\mathrm{g}}$, the conduction band bends downward at the metal/ semiconductor interface and the direction of the built-in electric field points from $\mathrm{MoS}_{2}$ to electrode. When $V_{\mathrm{g}}$ moves from positive to negative, the band bending direction changes from downward to upward and the built-in electric field reverses. The polarity of the photocurrent generated by PVE is dependent on the direction of the built-in electric field. However, in the PTE model, the generated photocurrent is equal to thermal voltage divided by device resistance $R$. Here, the thermal voltage is proportional to the local temperature increase in the junction, as well as to the difference in the Seebeck coefficients $(S)$ between the $\mathrm{MoS}_{2}$ and the electrode ${ }^{20}$. Herein, $S$ of the metal electrodes is negligible with respect to that of $\mathrm{MoS}_{2}{ }^{42}$; the latter is gate-dependent and given by the Mott relation ${ }^{19,43}$ as follows:

$$
S=\left.\frac{\pi^{2} k_{B}^{2} T}{3 q} \frac{1}{R} \frac{d R}{d V_{g}} \frac{d V_{g}}{d E}\right|_{E=E_{F}}
$$

where, $k_{\mathrm{B}}$ is the Boltzmann constant, $q$ is elementary charge, $T$ is absolute temperature, and $E_{\mathrm{F}}$ is Fermi energy. The calculated $S$ is plotted in Fig. 3d (black curve) using equation (1) in combination with the transfer characteristic curve of the device in dark. $S$ shows a non-monotonic variation with gate bias, maximum at the threshold voltage, $V_{\mathrm{t}}(\sim-10 \mathrm{~V})$ and reversing sign as the majority carrier changes from electron to hole when $V_{\mathrm{g}}<-15 \mathrm{~V}$. At $V_{\mathrm{g}}>0 \mathrm{~V}$, both PVE and PTE contributions have the same sign, and therefore yield a large photocurrent, as illustrated in Fig. 3c. However, in the regime where the built-in electric field just switches the sign, the two contributions can have opposite signs and the photocurrent can then have either sign depending on their relative strengths.

In order to gain more insights into the photocurrent generation mechanisms, we performed line-scan across the contact edge at a step size of $200 \mathrm{~nm}$ as shown by the black dashed line in Fig. 3a at various $V_{\mathrm{g}}$ (Figs. $4 \mathrm{a}-\mathrm{b}$ ). The peak position and peak amplitude of the photocurrents are extracted and plotted in Fig. 4c. The most striking 
a
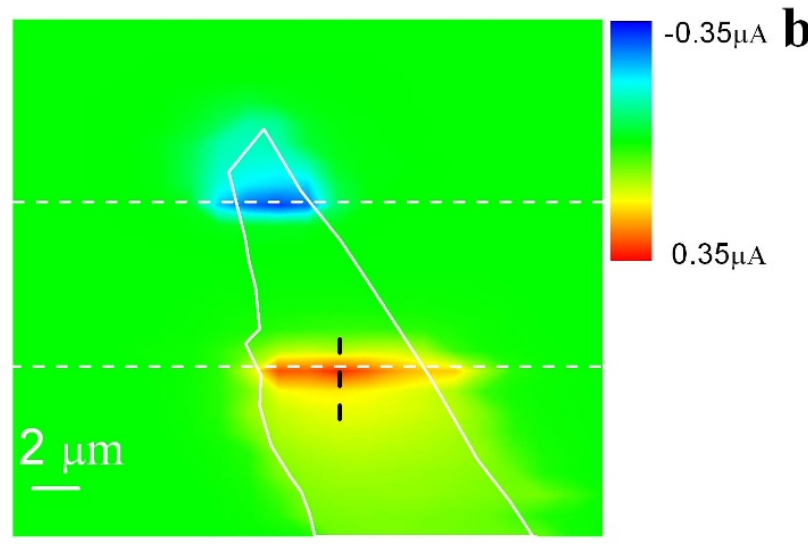

c
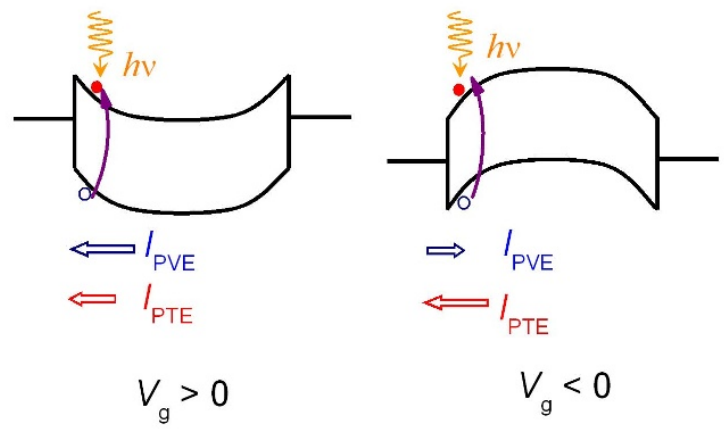
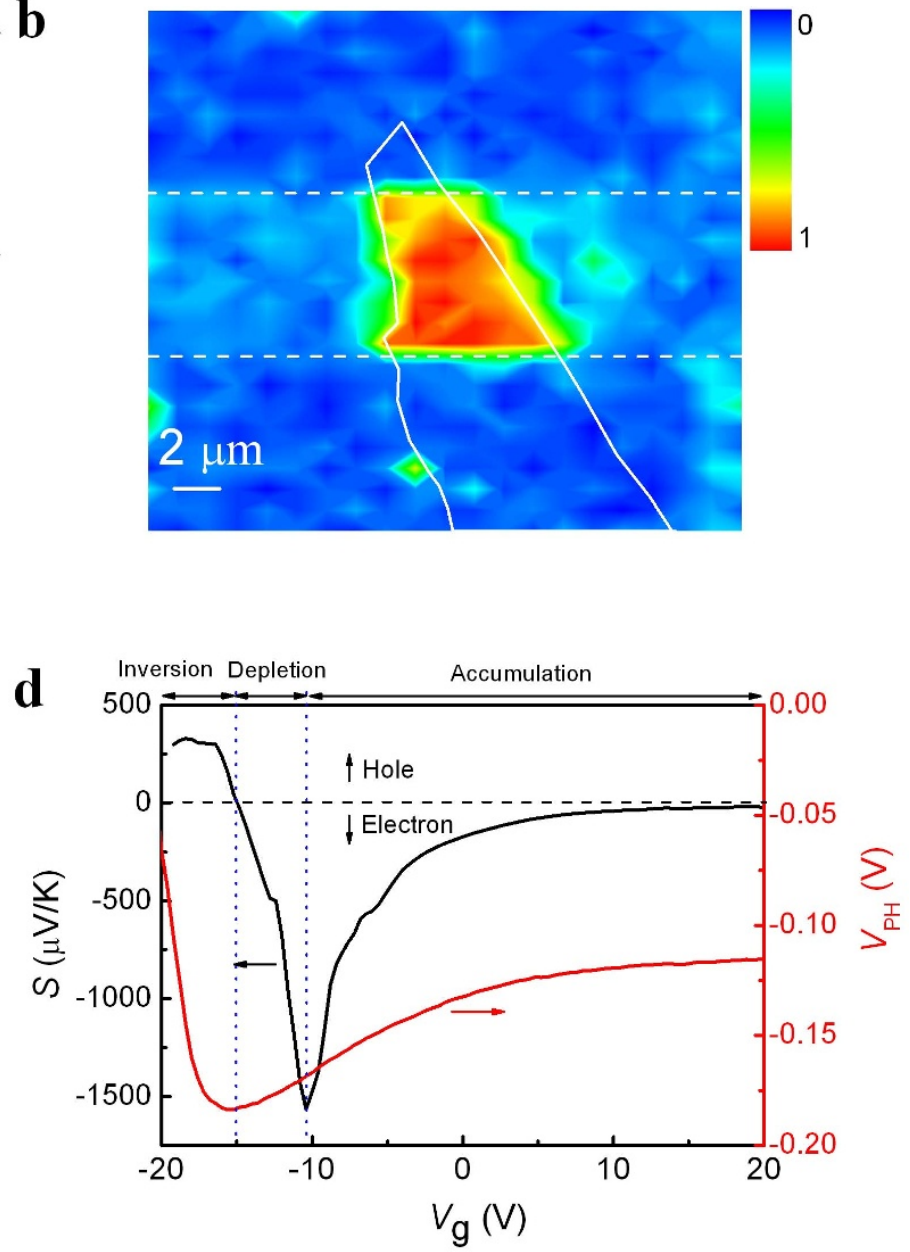

Figure 3 Photocurrent and Raman images of $\mathrm{MoS}_{2}$ transistor. (a) Short-circuit scanning photocurrent images taken at $V_{\mathrm{g}}=15 \mathrm{~V}$ with a laser power of $100 \mu \mathrm{W}$. (b) Raman mapping image with the normalized $\mathrm{E}_{2 \mathrm{~g}}^{1}$ peak intensity of the $\mathrm{MoS}_{2}$ multilayer. In panels (a) and (b), the dashed lines indicate the edges of the source and drain electrodes while the white lines mark the boundaries of $\mathrm{MoS}_{2}$. (c) Energy-band diagram of a multilayer- $\mathrm{MoS}_{2}$ transistor under light illumination in the accumulation $\left(V_{\mathrm{g}}>0 \mathrm{~V}\right)$ and depletion $\left(V_{\mathrm{g}}<0 \mathrm{~V}\right)$ regimes. Electron-hole pairs are generated in the spacecharge region by light absorption and contribute to PVE and PTE photocurrents, which are indicated by blue and red arrows, respectively. (d) Gatedependent Seebeck coefficient ( $S$, black line) and the open-circuit photovoltage ( $V_{\mathrm{PH}}$, red line) with a 100- $\mu \mathrm{W}$ laser illuminated at the contact edge. The vertical blue dotted lines separate the regimes of accumulation, depletion and inversion while the horizontal black dashed line indicates the border between the electron and hole conductions.

feature in the plot is the movement of the peak position at the contact edge as $V_{\mathrm{g}}$ is decreased below $-5 \mathrm{~V}$. Concurrently, the peak amplitude decreases exponentially with $V_{\mathrm{g}}$ without sign flip. According to Fig. $3 \mathrm{~d}$, the thermoelectric photocurrent is expected to be negligible in the depletion regime $\left(-15 \mathrm{~V}<V_{\mathrm{g}}<-10 \mathrm{~V}\right)$ since $S$ approaches zero in this region. As a result, PVE should dominate the photocurrent there. When $V_{\mathrm{g}}$ is changed from positive to negative, the built-in electric field reverses and the peak electric field at negative $V_{\mathrm{g}}$ moves away from the contact edge and extends more into the channel region compared to that at positive $V_{\mathrm{g}}$ (Supplementary Fig. S6). At $V_{\mathrm{g}}=-15 \mathrm{~V}$, the photocurrent peak moves $\sim 0.6 \mu \mathrm{m}$ away from the contacts, which is in qualitative agreement with the movement of the simulated peak electric field. If the photocurrent generation is dominated by PVE in the depletion regime, the sign of photocurrent should reverse when $\left(V_{\mathrm{g}}-V_{\mathrm{t}}\right)$ changes from positive to negative, which is however not observed experimentally. The contradicting polarity of the photocurrent suggests that the effect of the built-in field is less likely to be the dominant mechanism to determine the direction of the photocurrent in the depletion regime.

Thermoelectric power with two-carrier transport. Actually, the Mott formulism, which is established on the basis of thermal diffusion of one carrier type, is not valid for two types of carriers involved in the ambipolar transport. With light illumination, e-h pairs are generated when the photon energy exceeds the bandgap. Such excited carriers should contribute not only to the electrical conductivity, but also to the thermoelectric power through changing carrier concentration, which is more significant in the depletion regime. For such a two-carrier process, the thermoelectric power is given by ${ }^{44}$

$$
S=-\frac{k_{B}}{q} \frac{n \mu_{n}\left(A_{n}+\ln \frac{N_{c}}{n}\right)-p \mu_{p}\left(A_{p}+\ln \frac{N_{v}}{p}\right)}{n \mu_{n}+p \mu_{p}}
$$

where, $n$ and $p$ are electron and hole concentrations, respectively, $\mu_{\mathrm{n}}$ and $\mu_{\mathrm{p}}$ are the corresponding mobilities, and $N_{\mathrm{c}}$ and $N_{\mathrm{v}}$ are the effective density of states in the conduction band and valence band, respectively, and $A_{\mathrm{n}}$ and $A_{\mathrm{p}}$ are thermoelectric constants of electrons and holes. When the concentration of one type of carriers is much higher than that of the opposite type of carriers, such as in the accumulation regime, $S$ is then dominated by the majority carriers and equation (2) is reduced to the Mott relation ${ }^{43}$. If the electrons and the holes are equal in number, for example for photo-excited e-h 

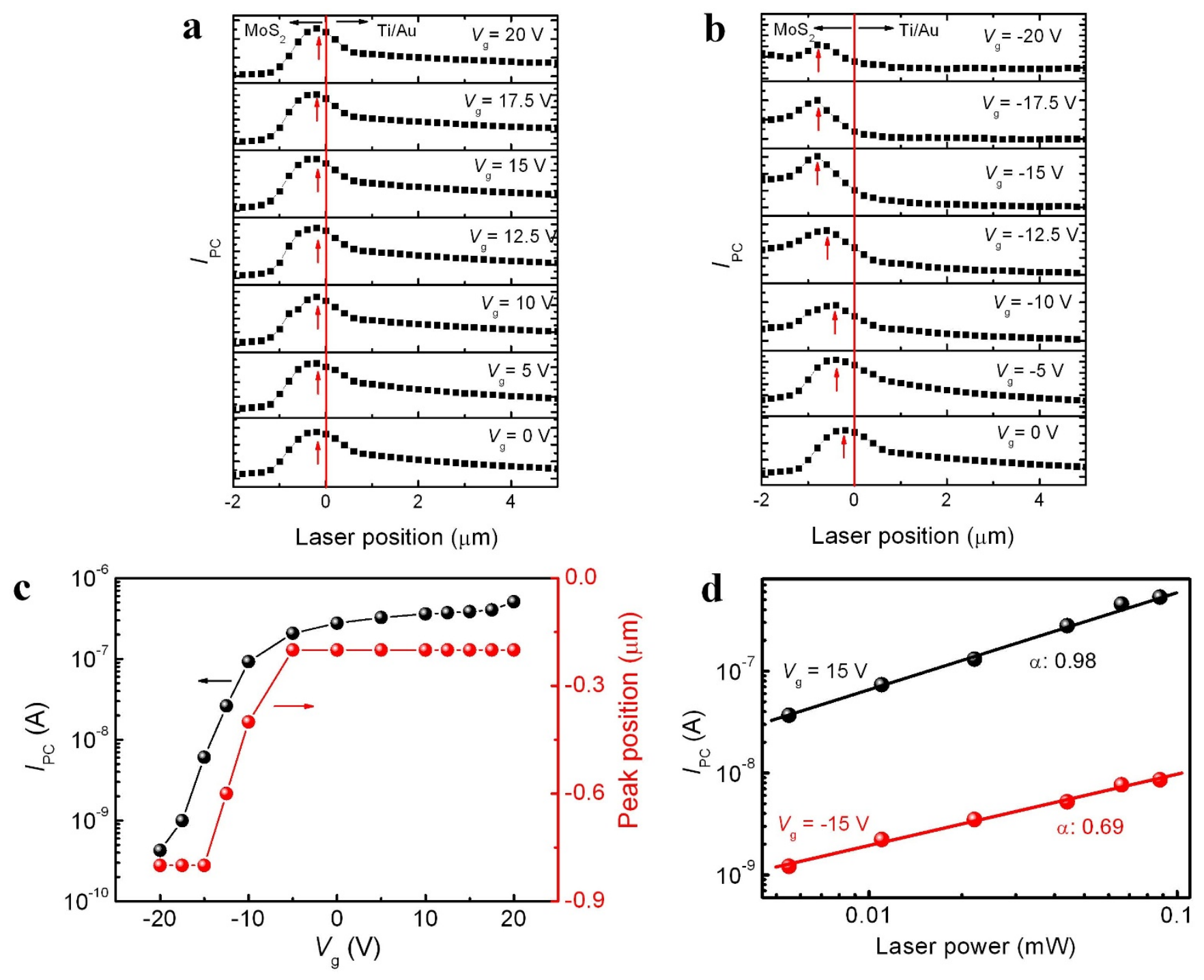

Figure $4 \mid$ Gate-dependent photocurrent in $\mathrm{MoS}_{2}$ transistor. (a) Photocurrent $\left(I_{\mathrm{PC}}\right)$ line scans across the contact edge as indicated by the black dashed line in Fig. $3 \mathrm{a}$ at $V_{\mathrm{g}}$ from $0 \mathrm{~V}$ to $20 \mathrm{~V}$ and (b) $V_{\mathrm{g}}$ from $0 \mathrm{~V}$ to $-20 \mathrm{~V}$. The red arrows in panels (a) and (b) indicate the position of peak photocurrent. (c) Photocurrent peak position and amplitude in panels (a) and (b) varied with $V_{\mathrm{g}}$. (d) The laser-power $(P)$ dependency of the photocurrent with laser illuminated at the contact edge for $V_{\mathrm{g}}=15 \mathrm{~V}$ and $-15 \mathrm{~V}$. The black and red lines are power-law fits with $I_{\mathrm{PC}} \propto P^{\alpha}$.

pairs in the depletion regime, the effective carrier density of states would play a dominant role in determining the amplitude and sign of thermoelectric power. In multilayer $\mathrm{MoS}_{2}$, the minimum of the conduction band lies in the lower symmetry Q point in the $k$-space along the $\Gamma$-K line with a 6 -fold valley degeneracy while the single valence band maximum is situated at the $\Gamma$ point ${ }^{3}$. This results in an effective density of states in the conduction band almost 6 times that in the valence band by assuming an equal effective mass for electrons and holes. Therefore, PTE in the multilayer $\mathrm{MoS}_{2}$ is still dominated by the photo-excited electrons and both $S$ and conductivity increase with illumination in the depletion regime. The unchanged polarity of photocurrent in the $\mathrm{MoS}_{2}$ FETs, when $V_{\mathrm{g}}$ is swept from positive to negative in Fig. 4b, reveals that PTE is predominant over PVE in the depletion regime. However, this photo-induced $S$ in $\mathrm{MoS}_{2}$ is spatially inhomogeneous along the channel. In the space charge region, the built-in electric field reduces the e-h recombination rate, resulting in a higher $S$ compared to that in the flat-band region. Recent studies ${ }^{22,45}$ reveal that a nonuniform $S$ in the channel could produce an additional PTE photocurrent, which is different from that at the semiconductor-metal junction. This PTE photocurrent, as observed in Fig. 4b, moves with the gate bias in the depletion regime due to the photo-induced $S$ depending on the electric field.
Hot photo-excited carrier inducing giant Seebeck coefficient. Apart from short-circuit photocurrent, open-circuit photovoltage can be a complementary diagnostic parameter to further probe the nature of the photoresponse. As shown in Fig. 3d (red curve), the gate-dependent photovoltage is measured at the contact edge. Identical to the photocurrent in Fig. 4c, the sign of photovoltage is not reversed during the gate sweeping and the maximum of photovoltage is shifted to more negative voltage $(-15 \mathrm{~V})$ compared to the gate-dependent $S$. Obviously, such a negative shift can be attributed to the photo-excited carriers. Since the main contribution of photovoltage arises from PTE in the depletion regime, the maximum $S$ can be estimated from the photovoltage because there is only a constant temperature difference in between them. We have already determined that approximately a temperature increase by $5 \mathrm{~K}$ is obtained with a $100-\mu \mathrm{W}$ laser illumination. The $-0.18 \mathrm{~V}$ peak photovoltage would correspond to an $S$ value of $-3.6 \times 10^{4} \mu \mathrm{V} / \mathrm{K}$, which is one order of magnitude larger than the calculated maximum $S$ value (black curve in Fig. 3d). This discrepancy in $S$ has also been reported by Buscema et al. ${ }^{23}$. Nonetheless, the $S$ value obtained for multilayer $\mathrm{MoS}_{2}$ is much larger than that of graphene ${ }^{19,20}$, carbon nanotubes $^{21,22}$ and other thermoelectric materials ${ }^{46,47}$. In the 
estimation of $S$ from the photovoltage data, the temperature increase induced by laser illumination is assumed to be equivalent to the temperature rise causing the Raman shift in the $\mathrm{MoS}_{2}$. However, this Raman-shift thermometry measures the phonon temperature instead of the electron temperature in the $\mathrm{MoS}_{2}$. It is the gradient in the electron, rather than phonon, temperature that ultimately drives the PTE effect. In the course of photo-excitation, the electrons and the phonons do not reach thermal equilibrium ${ }^{48}$. For example, in laser-illuminated graphene layers, electrons are much hotter than the surrounding crystal lattices and form a hot Fermion distribution ${ }^{19,49,50}$. Therefore, the measured phonon temperature is likely to underestimate the temperature of the electrons in the multilayer $\mathrm{MoS}_{2}$, resulting in a false anomalously giant $S$.

Strikingly, when $V_{\mathrm{g}}<-15 \mathrm{~V}$, the calculated $S$ in Fig. 3d reverses sign due to a weak hole inversion, while the polarity of photovoltage is opposite still staying positive. This suggests that the thermoelectric effect is still dominated by the excited hot electrons according to equation (2). In the strong electron accumulation regime $\left(V_{\mathrm{g}}>\right.$ $0 \mathrm{~V})$, the photovoltage saturates at a relative high value of $-0.12 \mathrm{~V}$, which results in a ratio of maximum-to- saturation photovoltage to be $\sim 1.5$. This is nearly two orders of magnitude smaller than that of calculated $S\left(\sim 10^{2}\right)$. This indicates that PVE rather than PTE is the dominant effect in the accumulation regime because the high electron concentration induced by gate voltage effectively cools the excited electrons due to the strong electron-electron interaction in reduced-dimension semiconductors ${ }^{51}$.

It has recently been demonstrated that the power-law relationship between photocurrent and incident laser power $(P)$, i.e. $I_{\mathrm{PC}} \propto P^{\alpha}$, can be used as an alternative approach to characterizing the dominant mechanism for photocurrent generation ${ }^{52}$. The exponent $\alpha$ is unity for PVE and 2/3 for PTE. The laser-power dependency of the photocurrent at $V_{\mathrm{g}}=15 \mathrm{~V}$ and $-15 \mathrm{~V}$ is shown in Fig. $4 \mathrm{~d}$ with the laser focused at the contact edge. The linear relationship at $V_{\mathrm{g}}=15 \mathrm{~V}$ and the sublinear dependence on the laser power $(\alpha=0.69)$ at $V_{\mathrm{g}}=$ $-15 \mathrm{~V}$ further confirm that PVE dominates in the accumulation while PTE dictates in the depletion. This observation strikingly contradicts the common perception that one photocurrent mechanism (PVE or PTE) should dominate in the whole working regime, as is the case for nanotubes ${ }^{21,22,37,38}$ and graphene ${ }^{19,20}$. That PTE rather than PVE dominates in the depletion regime can be explained as follows. The optical absorption of $\mathrm{MoS}_{2}$ creates hot electrons. They can gain sufficient kinetic energy to overcome the potential barrier in the depletion regime. As a result, the hot electrons thermally diffusing along the channel generate the observed photocurrent in the depletion regime.

\section{Discussion}

A spatially resolved photoresponse of multilayer-MoS2 transistors has been investigated by using SPCM. When exposed to ambient environment, the transistors are electrically unstable causing undesired hysteresis and persistent photoconductivity when they are characterized using conventional DC measurement. Such instabilities are completely eliminated by using a novel pulsedgate-bias method. As a result, a stable and repeatable photoresponse of $\mathrm{MoS}_{2}$ transistors is, for the first time, to our knowledge, achieved in ambient environment. This has allowed us to systematically explore the interplay of thermal, optical and electrical behaviors of multilayer $\mathrm{MoS}_{2}$. Both photovoltaic and photothermoelectric effects are found to be at work in the multilayer- $\mathrm{MoS}_{2}$ transistors, although they take dominant roles in different regimes of device operation. In the accumulation regime, the PVE at the $\mathrm{MoS}_{2}$-metal interface is the dominant mechanism whereas the hot-carrier-assisted PTE prevails in the depletion regime. This behavior is different from that observed in other low-dimensional materials that only contribute with either PVE or PTE. Moreover, the anomalously large Seebeck coefficient in $\mathrm{MoS}_{2}$, which has also been reported by others, is found to be the consequence of an oversimplification by assuming thermal equilibrium of the hot photo-excited carriers with the surrounding phonons. Our findings that shed light on the photoresponse mechanism of multilayer $\mathrm{MoS}_{2}$ may apply to other TMDs as well. They will also be helpful in advancing the art in light harvesting and photodetector applications.

\section{Methods}

Exfoliation and characterization of $\mathrm{MoS}_{2}$ thin flakes. Thin $\mathrm{MoS}_{2}$ flakes were peeled off from bulk $\mathrm{MoS}_{2}$ (SPI supplies) by mechanical exfoliation. They were subsequently transferred to a heavily doped p-type Si substrate with a 300 -nm-thick thermally grown $\mathrm{SiO}_{2}$. The $\mathrm{SiO}_{2} / \mathrm{Si}$ substrate was pre-cleaned by sonication in acetone, isopropyl alcohol, and deionized water. The transferred $\mathrm{MoS}_{2}$ flakes were identified using an optical microscope (Keyence digital microscope VHX-600). The thickness of the $\mathrm{MoS}_{2}$ flakes was measured using AFM (Dimension 3100 with Nanoscope IIIa controller, Veeco) operated in tapping mode under ambient conditions. Raman spectra of $\mathrm{MoS}_{2}$ films were acquired by employing micro-Raman spectrometry (Renishaw inVia) in a backscattering configuration with a He-Ne $633 \mathrm{~nm}$ laser. The scattered signal was collected through a $100 \times$ objective and dispersed by a grating of 1800 lines/mm with a spectral resolution of $\sim 1.0 \mathrm{~cm}^{-1}$ and a peak position accuracy of $0.1 \mathrm{~cm}^{-1}$. The power of the excitation laser line was kept well below $0.1 \mathrm{~mW}$ to avoid sample heating and an exposure time of $30 \mathrm{~s}$ was used to improve the signal-tonoise ratio.

Device fabrication and electrical characterization. In order to avoid the contamination from photolithography or electron-beam lithography, a $10-\mu \mathrm{m}$ spacing copper grid was placed on top of the thin $\mathrm{MoS}_{2}$ flakes as a shadow mask for the electrode fabrication. A bilayer stack $\mathrm{Ti} / \mathrm{Au}$ of $5 / 50 \mathrm{~nm}$ thickness was then deposited by means of electron-beam evaporator as the source and drain electrodes. Heavily doped Si substrate was used as the common back gate for the fabricated $\mathrm{MoS}_{2}$ FETs. Electrical characterization of the devices was carried out in a shielded probe station with Keithley 4200 semiconductor characterization system in the ambient environment.

SPCM characterization. Spatial dependence of photoresponse in the $\mathrm{MoS}_{2}$ transistors was obtained on the Renishaw Raman spectrometer equipped with the same electrical characterization system described above. A laser beam of 633-nm wavelength was focused to a diffraction-limited spot size of about $0.6 \mu \mathrm{m}$ by using a long-working distance $50 \times$ objective lens. The incident laser power could be adjusted with the use of neutral density filters. A computer-controlled XYZ translational stage was used to acquire scanning photocurrent image and Raman mapping image simultaneously. When the laser spot was raster-scanned over the device area, the resultant current flow between electrodes and the Raman spectrum for each point in the scan were recorded simultaneously, thus generating spatially resolved images of photocurrent and Raman signal. All scanning photocurrent measurements were performed at room temperature in air.

1. Xu, M., Liang, T., Shi, M. \& Chen, H. Graphene-like two-dimensional materials Chem. Rev. 113, 3766-3798 (2013).

2. Schwierz, F. Graphene transistors. Nature Nanotech. 5, 487-496 (2010).

3. Wang, Q. H., Kalantar-Zadeh, K., Kis, A., Coleman, J. N. \& Strano, M. S. Electronics and optoelectronics of two-dimensional transition metal dichalcogenides. Nature Nanotech. 7, 699-712 (2012).

4. Chhowalla, M. et al. The chemistry of two-dimensional layered transition metal dichalcogenide nanosheets. Nature Chem. 5, 263-275 (2013).

5. Mak, K. F., Lee, C., Hone, J., Shan, J. \& Heinz, T. F. Atomically thin $\mathrm{MoS}_{2}$ : a new direct-gap semiconductor. Phys. Rev. Lett. 105, 136805 (2010).

6. Splendiani, A. et al. Emerging photoluminescence in monolayer $\mathrm{MoS}_{2}$. Nano Lett. 10, 1271-1275 (2010)

7. Radisavljevic, B., Radenovic, A., Brivio, J., Giacometti, V. \& Kis, A. Single-layer $\mathrm{MoS}_{2}$ transistors. Nature Nanotech. 6, 147-150 (2011).

8. Kim, S. et al. High-mobility and low-power thin-film transistors based on multilayer $\mathrm{MoS}_{2}$ crystals. Nature Commun. 3, 1011 (2012).

9. Lopez-Sanchez, O., Lembke, D., Kayci, M., Radenovic, A. \& Kis, A. Ultrasensitive photodetectors based on monolayer $\mathrm{MoS}_{2}$. Nature Nanotech. 8, 497-501 (2013).

10. Choi, W. et al. High-detectivity multilayer $\mathrm{MoS}_{2}$ phototransistors with spectral response from ultraviolet to infrared. Adv. Mater. 24, 5832-5836 (2012).

11. Li, H. et al. Fabrication of single- and multilayer $\mathrm{MoS}_{2}$ film-based field-effect transistors for sensing NO at room temperature. Small 8, 63-67 (2012).

12. Castellanos-Gomez, A. et al. Electric-field screening in atomically thin layers of $\mathrm{MoS}_{2}$ : the role of interlayer coupling. Adv. Mater. 25, 899-903 (2013).

13. Kaasbjerg, K., Thygesen, K. S. \& Jacobsen, K. W. Phonon-limited mobility in ntype single-layer $\mathrm{MoS}_{2}$ from first principles. Phys. Rev. B 85, 115317 (2012).

14. Yin, Z. et al. Single-layer $\mathrm{MoS}_{2}$ phototransistors. ACS Nano 6, 74-80 (2012).

15. Korn, T., Heydrich, S., Hirmer, M., Schmutzler, J. \& Schüller, C. Low-temperature photocarrier dynamics in monolayer $\mathrm{MoS}_{2}$. Appl. Phys. Lett. 99, 102109 (2011).

16. Shi, H. et al. Exciton dynamics in suspended monolayer and few-layer $\mathrm{MoS}_{2} 2 \mathrm{D}$ crystals. ACS Nano 7, 1072-1080 (2013). 
17. $\mathrm{Wu}$, C.-C. et al. Elucidating the photoresponse of ultrathin $\mathrm{MoS}_{2}$ field-effect transistors by scanning photocurrent microscopy. J. Phys. Chem. Lett. 4, 2508-2513 (2013).

18. Li, H.-M. et al. Metal-semiconductor barrier modulation for high photoresponse in transition metal dichalcogenide field effect transistors. Sci. Rep. 4, 4041 (2014).

19. Gabor, N. M. et al. Hot carrier-assisted intrinsic photoresponse in graphene. Science 334, 648-652 (2011).

20. Xu, X., Gabor, N. M., Alden, J. S., van der Zande, A. M. \& McEuen, P. L. Photothermoelectric effect at a graphene interface junction. Nano Lett. 10, 562-566 (2010).

21. St-Antoine, B. C., Ménard, D. \& Martel, R. Position sensitive photothermoelectric effect in suspended single-walled carbon nanotube films. Nano Lett. 9, 3503-3508 (2009).

22. DeBorde, T., Aspitarte, L., Sharf, T., Kevek, J. W. \& Minot, E. D.

Photothermoelectric effect in suspended semiconducting carbon nanotubes. ACS Nano 8, 216-221 (2014).

23. Buscema, M. et al. Large and tunable photothermoelectric effect in single-layer $\mathrm{MoS}_{2}$. Nano Lett. 13, 358-363 (2013).

24. Novoselov, K. S. et al. Electric field effect in atomically thin carbon films. Science 306, 666-669 (2004)

25. Li, H. et al. From bulk to monolayer $\mathrm{MoS}_{2}$ : evolution of Raman scattering. $A d v$. Funct. Mater. 22, 1385-1390 (2012).

26. Kim, W. et al. Hysteresis caused by water molecules in carbon nanotube fieldeffect transistors. Nano Lett. 3, 193-198 (2003).

27. Levesque, P. L. et al. Probing charge transfer at surfaces using graphene transistors. Nano Lett. 11, 132-137 (2011).

28. Qu, M., Li, H., Liu, R., Zhang, S.-L. \& Qiu, Z.-J. Interaction of bipolaron with the $\mathrm{H}_{2} \mathrm{O} / \mathrm{O}_{2}$ redox couple causes current hysteresis in organic thin-film transistors. Nature Commun. 5, 3185 (2014).

29. Late, D. J., Liu, B., Ramakrishna Matte, H. S. S., Dravid, V. P. \& Rao, C. N. R. Hysteresis in single-layer $\mathrm{MoS}_{2}$ field effect transistors. ACS Nano 6, 5635-5641 (2012)

30. Cho, K. et al. Electric stress-induced threshold voltage instability of multilayer $\mathrm{MoS}_{2}$ field effect transistors. ACS Nano 7, 7751-7758 (2013).

31. Mattmann, M. et al. Pulsed gate sweep strategies for hysteresis reduction in carbon nanotube transistors for low concentration $\mathrm{NO}_{2}$ gas detection. Nanotechnology 21, 185501 (2010).

32. Liu, Z., Qiu, Z.-J., Zhang, Z.-B., Zheng, L.-R. \& Zhang, S.-L. Mobility extraction for nanotube TFTs. IEEE Elec. Dev. Lett. 32, 913-915 (2011).

33. Zhang, W. et al. High-gain phototransistors based on a CVD $\mathrm{MoS}_{2}$ monolayer. Adv. Mater. 25, 3456-3461 (2013).

34. Radisavljevic, B. \& Kis, A. Mobility engineering and metal-insulator transition in monolayer $\mathrm{MoS}_{2}$. Nature Mater. 12, 815-820 (2013).

35. Castellanos-Gomez, A. et al. Laser-thinning of $\mathrm{MoS}_{2}$ : on demand generation of a single-layer semiconductor. Nano Lett. 12, 3187-3192 (2012).

36. Ahn, Y., Dunning, J. \& Park, J. Scanning photocurrent imaging and electronic band studies in silicon nanowire field effect transistors. Nano Lett. 5, 1367-1370 (2005).

37. Freitag, M. et al. Imaging of the Schottky barriers and charge depletion in carbon nanotube transistors. Nano Lett. 7, 2037-2042 (2007).

38. Balasubramanian, K., Burghard, M., Kern, K., Scolari, M. \& Mews, A. Photocurrent imaging of charge transport barriers in carbon nanotube devices. Nano Lett. 5, 507-510 (2005)

39. Sanders, A. W. Optical properties of metallic nanostructures. Ph.D. thesis, Yale University, New Haven, Connecticut, 2007.

40. Yan, R. et al. Thermal conductivity of monolayer molybdenum disulfide obtained from temperature-dependent Raman spectroscopy. ACS Nano 8, 986-993 (2014).

41. Sahoo, S., Gaur, A. P. S., Ahmadi, M., Guinel, M. J.-F. \& Katiyar, R. S. Temperature-dependent Raman studies and thermal conductivity of few-layer $\mathrm{MoS}_{2}$. J. Phys. Chem. C 117, 9042-9047 (2013)
42. Cusack, N. \& Kendall, P. The absolute scale of thermoelectric power at high temperature. Proc. Phys. Soc. 72, 898-901 (1958).

43. Ashcroft, N. W. \& Mermin, N. D. Solid State Physics Ch. 13 (Thomson Learning Inc., London, 1976)

44. Harper, J. G., Matthews, H. E. \& Bube, R. H. Photothermoelectric effects in semiconductors: n- and p-type silicon. J. Appl. Phys. 41, 765-770 (1970)

45. Nanot, S. et al. Broadband, polarization-sensitive photodetector based on optically-thick films of macroscopically long, dense, and aligned carbon nanotubes. Sci. Rep. 3, 1335 (2013).

46. Ohta, H. et al. Giant thermoelectric seebeck coefficient of a two-dimensional electron gas in $\mathrm{SrTiO}_{3}$. Nature Mater. 6, 129-134 (2007)

47. Pernstich, K. P., Rössner, B. \& Batlogg, B. Field-effect-modulated Seebeck coefficient in organic semiconductors. Nature Mater. 7, 321-325 (2008).

48. Eesley, G. L. Generation of nonequilibrium electron and lattice temperatures in copper by picosecond laser pulses. Phys. Rev. B 33, 2144-2151 (1986).

49. Sun, D. et al. Ultrafast hot-carrier-dominated photocurrent in graphene. Nature Nanotech. 7, 114-118 (2012).

50. Song, J. C. W., Rudner, M. S., Marcus, C. M. \& Levitov, L. S. Hot carrier transport and photocurrent response in graphene. Nano Lett. 11, 4688-4692 (2011).

51. Liang, Y., Huang, S., Soklaski, R. \& Yang, L. Quasiparticle band-edge energy and band offsets of monolayer of molybdenum and tungsten chalcogenides. Appl. Phys. Lett. 103, 042106 (2013)

52. Patil, V., Capone, A., Strauf, S. \& Yang, E.-H. Improved photoresponse with enhanced photoelectric contribution in fully suspended graphene photodetectors. Sci. Rep. 3, 279 (2013).

\section{Acknowledgments}

This work is supported partially by the Special Funds for Major State Basic Research Projects of China (No. 2011CBA00603), National Natural Science Foundation of China (Nos. 61204090 and 61171010), Shanghai Municipal Natural Science Foundation (No. 12ZR1402700), Fundamental Research Project of young teachers to enhance research capacity, Fudan University (No. 20520133248), and the Knut \& Alice Wallenberg Foundation for a generous denotation to enable the "Swedish Graphene Initiative" project

\section{Author contributions}

Z.-J.Q. conceived the research. Z.-J.Q. and Y.Z. designed the experiments. H.W., X.X. and YZ. carried out $\mathrm{MoS}_{2}$ device fabrication. Y.Z. performed AFM analysis, Raman measurement and SPCM characterizations. H.L. and L.W. performed TCAD simulation and first-principle calculations, respectively. Z.-J.Q. and Y.Z. analyzed the data. Z.-J.Q., S.-L.Z. and R.L. wrote the manuscript. All authors discussed the results and commented the manuscript.

\section{Additional information}

Supplementary information accompanies this paper at http://www.nature.com/ scientificreports

Competing financial interests: The authors declare no competing financial interests.

How to cite this article: Zhang, Y. et al. Photothermoelectric and photovoltaic effects both present in $\mathrm{MoS}_{2}$. Sci. Rep. 5, 7938; DOI:10.1038/srep07938 (2015).

This work is licensed under a Creative Commons Attribution-NonCommercialNoDerivs 4.0 International License. The images or other third party material in this article are included in the article's Creative Commons license, unless indicated otherwise in the credit line; if the material is not included under the Creative Commons license, users will need to obtain permission from the license holder in order to reproduce the material. To view a copy of this license, visit http:// creativecommons.org/licenses/by-nc-nd/4.0/ 